\title{
Enabling Cognition on Electronic CounterMeasure Systems against Next-Generation Radars
}

\author{
Muharrem Arik Ozgur Baris Akan \\ Next-generation and Wireless Communications Laboratory (NWCL) \\ Department of Electrical and Electronics Engineering \\ Koc University, Istanbul, Turkey \\ Email: \{marik, akan\}@ku.edu.tr
}

\begin{abstract}
Current Electronic CounterMeasure (ECM) systems process preprogrammed jamming techniques against radar threats that are captured and identified by Electronic Support Measures (ESM) systems. On the other side, with recent technologies, radar systems become adaptive and intelligent systems that can change their waveforms. Nevertheless, on-going studies in the radar domain have enabled cognition. However, an ECM architecture is yet to be developed for automatically generating effective countermeasures against new, unknown and next-generation radars. To address this need, enabling a cognitive control mechanism in the ECM system is a suitable solution. In this paper, we present an architecture for Cognitive Electronic CounterMeasure (CECM) system. This CECM system assesses the most effective ECM technique against new, unknown and adaptive or cognitive radars. Simulations reveal that CECM system provides accurate mapping performance while providing satisfying generalization for unknown emitters.
\end{abstract}

\section{INTRODUCTION}

Years ago, intruding vehicles passing through enemy area were able to approach only under cover of darkness, fog or rain. Radar technology made all efforts to camouflage the intrusion ineffective.

Several methods are still being used to overcome radar systems. One effective method is making the approach at low altitudes with a very low detection profile. The other method is reducing the reflected radar signal power. This method is accomplished by using special techniques and absorbing covering material to reduce the radar reflective area [1].

The last method to conceal the passing vehicle from enemy radar is Electronic CounterMeasure (ECM). Any intentional electronic attempt to confuse radar operation is generally referred as an ECM technique. There are two types of ECM, active and passive. Passive ECM methods involve radar absorbing material (RAM) and chaff, which is composed of a large number of small RF reflectors that provide large RCS. Active ECM systems are designed to inject disturbing, interfering or deceptive signals to radar receiver circuitry to prevent detection, locate and track protected vehicles [1].

To confuse enemy radar, a jamming signal can be a replica of the true reflected signal with amplitude, phase or frequency modulation. Fig. 1 shows a typical ECM system architecture. This kind of system is capable of executing preprogrammed ECM against different radars in various modes.

In Fig. 1, a receiver is included in the ECM system. This receiver is used to detect, analyse and classify all receiving signals to accurately determine which signals are associated with which radar in which mode. Analysis must be supported with angle of arrival information to successfully direct the transmitted jamming signal to corresponding enemy radar. Using an appropriate control mechanism on Digital RF Memory (DRFM) and the signal or noise source, various types of jamming techniques can be applied on a pulse-to-pulse basis. In addition, an amplitude/phase modulator is used to generate deceptive modulation on an intercepted signal to confuse the victim radar.

Current airborne Electronic Warfare (EW) systems must first evaluate enemy radar from its received signals to accurately assess the appropriate preprogrammed ECM technique. Especially in fighter jets and helicopters, there is no EW operator on-board to select or change the appropriate technique in operation. Therefore selection should be made by the system itself.

The preprogrammed ECM approach becomes less and less effective as radars evolve from fixed analogue systems to programmable digital variants, especially when adaptive or cognitive radars come to the battlefield. In the near future, these types of radars will be capable of sensing the environment and adapting their transmissions to increase performance and overcome jamming effects. Therefore, they will likely present greater challenges for current ECM systems.

In [2], an adaptive closed loop control solution for ECM systems was presented. In nature, ECM systems have to operate under some uncertainties as radar type or exact radar mode. Therefore, ECM systems mainly have to trust their threat radar database. In [2], an adaptive controller with system identification is proposed against tracking radar to overcome uncertainties about the threat. However, this solution is limited to certain ECM techniques against certain types of radar. Moreover, each system identification process for each radar should be independent; hence, an adaptive process must be initialized from the beginning state.

The Defence Advanced Research Projects Agency (DARPA) started a program called Adaptive Radar Countermeasure (ARC). The goal of this program is to enable U.S. airborne EW systems to automatically generate effective countermeasures against new, unknown and adaptive radars in real-time with minimum modification on current ECM systems in the field [7]. 


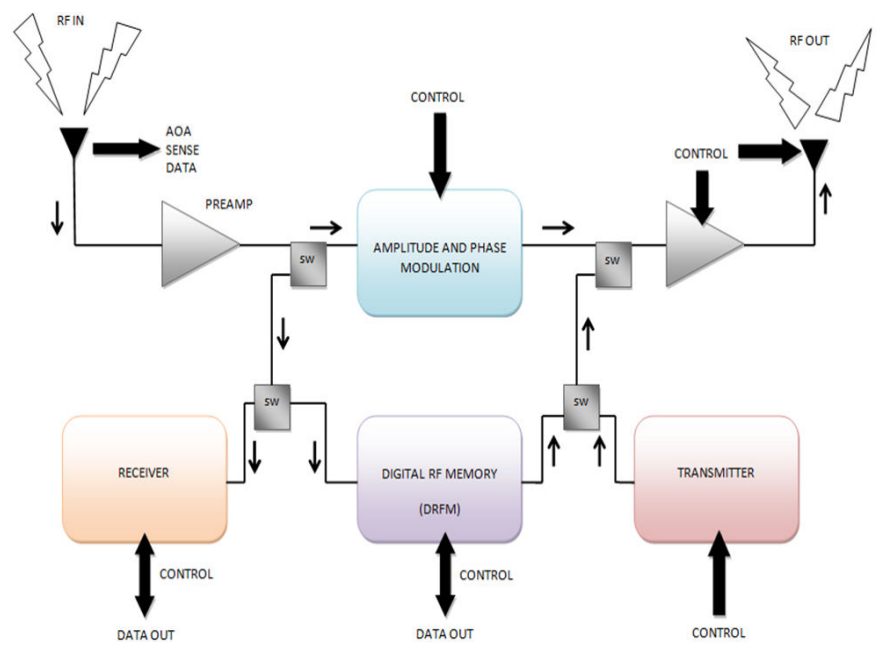

Fig. 1. Traditional ECM Architecture

The system scenarios being used in ECM systems is limited to fixed algorithms which may become obsolete in the near future, if the number of cognitive radar systems grows in the field. The ability to learn adapting to different situations must be included in the future design of ECM systems.

In this paper, we introduce an architecture for a Cognitive Electronic CounterMeasure (CECM) system. In our ECM architecture, the ability of adapting to different situations of the ECM system is achieved using cognitive capability. The CECM system has a unique technique selection architecture for unknown emitters using its generalization capability.

The remainder of the paper is organized as follows. Enabling cognition mechanism is presented in Section II. In Section III, we introduce the proposed cognitive structure and explain the architecture of the CECM. In Section IV, we present the performance evaluation and discuss the results. Finally, we give concluding remarks and future work in Section V.

\section{ENABLING COGNITION}

In this Section, we introduce an analogy between visual brain and ECM structure to enable cognition mechanism.

According to "dictionary.com", cognition is "the knowledge that results from such an act or process". The visual brain is a cognitive machine which has a inherent capability to accomplish certain missions as target tracking [6]. From an ECM system perspective, the functions of five fundamental building blocks in Fuster's paradigm of cognition are described below.

\section{A. Perception-Action Cycle}

The function of the perception-action cycle is to gather information about the environment using the received signal with the increasing information gain from one cycle to the next [6].
The main principle of the ECM system is reacting to a preprogrammed act according to the radar changing behaviour as a closed loop system. One analog for this system is sensing the RF environment via perception. Another is generating jamming signal via action. Furthermore, ECM systems already have a feedback mechanism between transmitter and receiver side to track the threat radar signal.

\section{B. Memory}

Memory extracts the features of the received radar signal to map the corresponding ECM technique. Memory stores the encoded information in its weights and parameters, and then recalls this information when needed. A Learning process is performed in the memory.

\section{Attention}

The function of attention is to provide the effective and efficient utilization of computational resources. When the effectiveness of the countermeasure is low in a PerceptionAction Cycle (PAC), selecting the optimum technique from the database requires an explore-exploit mechanism which may require high computational complexity. To avoid that complexity, a particular grid point in the ECM database is selected to closely match the feedback information on the preceding PAC. Therefore, the complexity enclosed in a global search of the entire database is substituted with a local search based on a small neighbour of grid points.

\section{Intelligence}

Intelligence empowers an algorithmic decision-making mechanism in the cognitive systems to intentional selection of a strategy for optimal solution of envisioned goal [6]. Specifically, enabling intelligence for optimal selection can reach a desired technique in the ECM system. Intelligence should be distributed throughout the ECM system.

\section{E. Language}

Finally, language is intended to provide effective and efficient communication on a person-to-person basis as well as a group of persons. However, we keep language out of scope of the cognitive ECM structure.

\section{Cognitive Electronic CounterMeasure (CECM) ARCHITECTURE}

An analogy between visual brain and ECM structure guides us to constitute a cognition enabled ECM structure to automatically generate effective countermeasures against new, unknown and cognitive radars in the field. To detect, deceive, and defeat enemy radar threats, current ECM systems must rely on known emitter databases to characterize the threat and determine the appropriate countermeasures response.

Cognition enables the ECM system to adaptively learn to defeat all potential threats using the advances in signal processing and machine learning to develop a system that detects and counters emerging radar threats. With this new technology, the Cognitive ECM system combines and transmits a countermeasure signal to reach a desired effect on the 


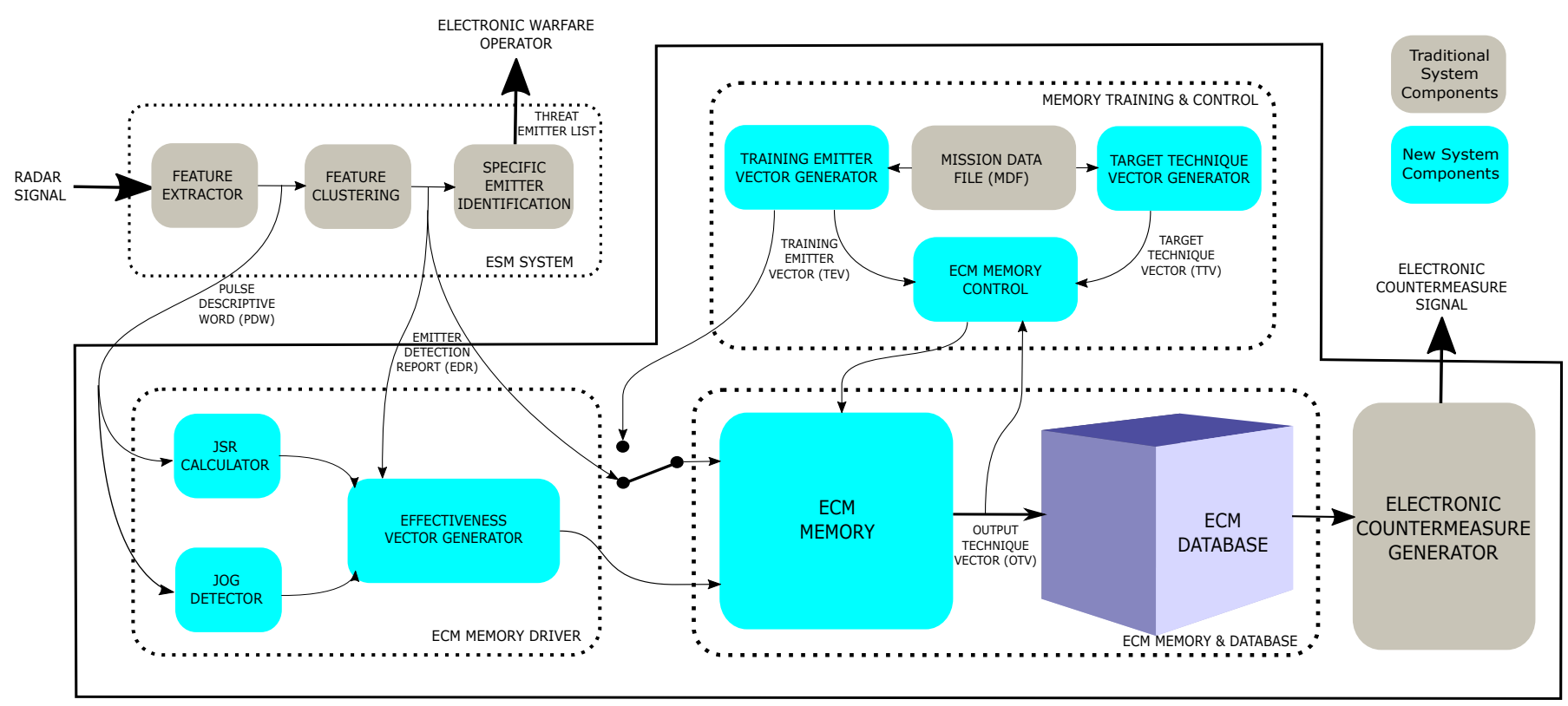

Fig. 2. Cognitive ECM Architecture

threat radar. Using a cost function, it assesses the effectiveness of countermeasures based on over-the-air observable threat behaviours.

The CECM system consists of three fundamental parts:

The ECM Memory \& Database is the first and vital part of the CECM system; the second is Memory Training \& Control; the ECM Memory Driver is the third part and generates feedback information required for optimization of the technique selection mechanism. After selecting a corresponding technique using memory, the ECM generator is responsible for the realization of the ECM technique vector. It consists a various ECM components such as Digital RF Memory (DRFM), AM \& PM Modulator and Noise Source.

\section{A. Feeding CECM System: Electronic SupportMeasures (ESM)}

Before selecting an effective countermeasure technique, the CECM system requires accurate detection and identification of threat emitter environment around the deployed platform. This information can be provided by the ESM system.

The ESM System has three blocks to reach the required information in Fig. 2. First, important features of gathered radar signals from the environment are extracted. These features help the ESM system detect and identify threat, neutral and unknown emitters. In the literature, different feature extraction methods have been proposed over the years [9], [10], [11].

In CECM, the ESM system supplies the signal's frequency, pulse width (PW) and time of arrival (ToA) information that packed in a pulse descriptive word (PDW). The Feature Extractor block provides PDWs to the second block called "feature clustering" or "deinterleaving". Using clustering algorithms, the second block detects radar emitter existence in the environment, then generates emitter detection reports (EDR) which include specific parameters as maximum and minimum frequency, PW, Pulse Repetition Interval (PRI), Antenna Rotation Type (ART) and Antenna Rotation Period (ARP). Finally, EDRs are used for emitter classification to specify the threat level of the emitter and the type of radar in the specific emitter identification block. Then, the threat emitter list is generated to show EW operator.

\section{B. ECM Memory \& Database}

This is the core and important part of the CECM system. The mission is mapping the most effective countermeasures from the ECM database to the known or unknown emitters.

At the input end of an ECM memory, there is EDR space, and at the output end, the ECM technique library. This library is composed of a grid of points with a layered structure. Each layer represents a different ECM technique space with a different combination of technique parameters, such as noise bandwidth, modulation depth, time or frequency offset, start or stop frequency, frequency step size or step visit duration. These parameters utilize ECM technique effectiveness against threat emitters.

To realize the ECM memory, a multilayer feed-forward neural network with sigmoid hidden neurons and linear output neurons is selected with two different structures as in Fig. 3. These neural networks fit multi-dimensional mapping problems well with the given consistent data and enough neurons in its hidden layers.

The idea behind this kind of neural network structure is referred to as features of features. Using the learning process, the first layer extracts the important features of the EDR. These features act as the input to the second layer and continues to extract the features of features of the original EDR.

The activation functions of neurons differ from layer to layer. For the second, third and fourth hidden layers, the 


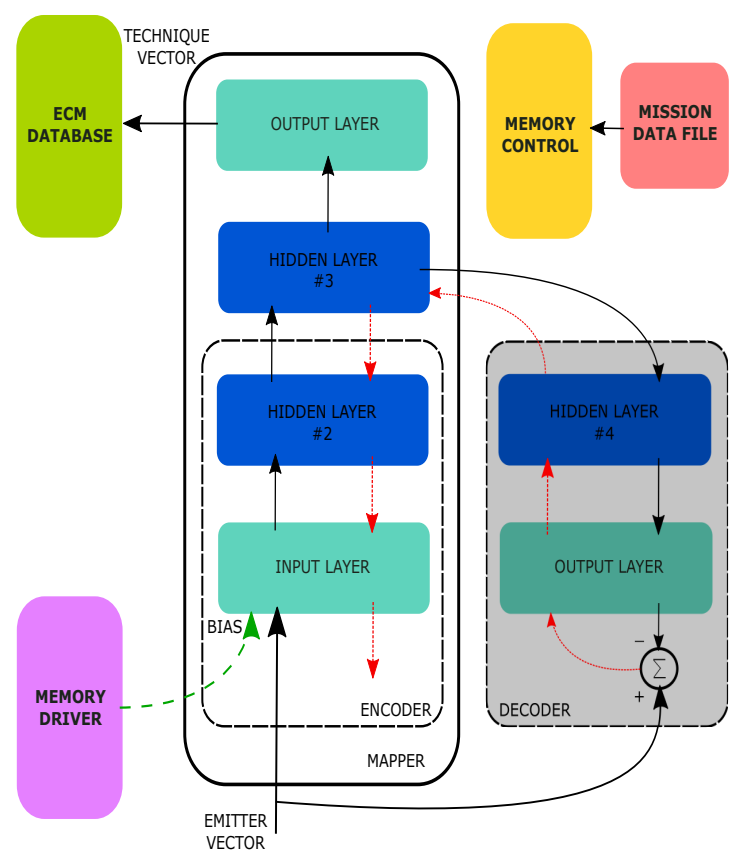

(a)

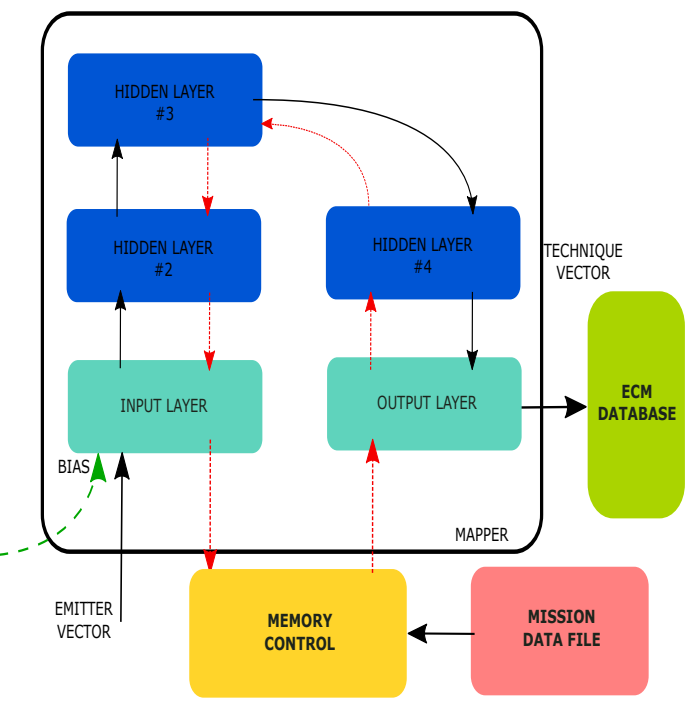

(b)

Fig. 3. Block Diagram of (a) Hybrid and (b) Multi-layer ECM Memory

activation functions are defined by the hyperbolic tangent function,

$$
\varphi^{(2)}=\varphi^{(3)}=\varphi^{(4)}=\tanh (\nu)
$$

with $\nu$ as the input to a neuron in a particular layer. For the output (fifth) layer, we use a linear activation function defined by,

$$
\varphi^{(5)}=\nu
$$

\section{Memory Training \& Control}

We propose two different types of memory structures called Hybrid and Multi-scale ECM Memory. The performance of these structures will be presented in Section IV.

Hybrid memory is shaped as two different neural network structures in Fig 3(a). The First is a replicator network, which is a simple choice of implementing the multi-scale memory [3]. Training of the replicator network is managed in an unsupervised fashion using the Bayesian Regularization Back-propagation algorithm in batch mode [4]. The Bayesian Regularization method minimizes the combination of squared errors and weights. This method also changes the linear combination to reach a good generalization specification at the end of training [12].

In the batch mode of unsupervised learning, adjustments to the weights of each layer are calculated after using all the examples in the training sample, which constitute one epoch of training [3]. Further, the Bayesian Regularization algorithm is selected to constitute the sufficient generalization of the network. The Training Emitter Vector (TEV) is applied to both the input layer and the output layer; hence, the emitter vector also takes the role of a desired response.

The second part is in a combined structure. Incorporating the encoder part of the replicator network and an additional output layer builds the combinational neural network. Once the training of replicator network is finished, the decoder part is no longer used. All weights in the encoder part are fixed, and then the hybrid network is trained under supervision of the Mission Data File (MDF) in batch mode. Using the MDF, TEVs and Training Technique Vectors (TTVs) are generated and used for the Least Mean Square (LMS) algorithm in the training process [3].

Multi-scale ECM memory is general type of multi-scale neural network structure in Fig 3(b). Training of the multiscale network is managed using the LMS algorithm under supervision of the MDF in batch mode [4].

Algorithmically, we use supervised training based on the LMS algorithm, described as, [3]

$$
\hat{\mathbf{w}}(n+1)=\hat{\mathbf{w}}(n)+\eta \mathbf{x}(n) e(n)
$$

where $\eta$ is the learning-rate parameter and $e(n)$ is the error signal, defined by,

$$
e(n)=d(n)-\mathbf{x}^{T}(n) \hat{\mathbf{w}}(n)
$$

where $d(n)$ is the teacher signal and $\mathbf{x}(n)$ is the input to the encoder in the replicator network.

It is important that the learning-rate parameter $\eta$ behave as a measure of the memory in the LMS algorithm. The smaller $\eta$, the longer the memory span over which the LMS algorithm remembers past data will be. Therefore, when $\eta$ is small, the 
LMS algorithm performs accurately, however the convergence rate of the algorithm is slow [3].

After finding the best technique vector, which might not be the same as in the MDF, on-line adaptation is required for the ECM memory. In the on-line mode of supervised learning, adjustments to the weights of each layer are performed on an example-by-example basis.

\section{ECM Memory Driver}

The driver extracts the ECM effectiveness information from the received radar signal behaviour. The first method is jog detection. Jog detection involves measuring the radar received power at the tracked platform, and searches for dips or jogs that happen when the radar antenna points away from the CECM deployed platform.

Jog detection is almost as old as tracking radars. However, multipath is a serious problem when searching jogs in the received signal, because signal power variations caused by multipath can be confused with jogs caused by the angle deception ECM. Several methods provide multipath and jog discrimination. In [8], an ECM system with two vertically separated antenna is proposed and detailed. Using the spatial diversity technique, the proposed system reliably detects jogs caused by the ECM system.

Although jog detection is used as an ECM effectiveness assessment metric, in our solution this phenomena drives our ECM memory as a bias vector to reach the most effective ECM technique parameters over the ECM Database.

Another mechanism to determine the success of the ECM signal is calculating of Jamming to Signal Ratio (JSR). Depending on the jamming type, emitter signal behaviour and distance to threat emitter, the JSR calculation gives an a priori information about the effectiveness of the ECM operation. In the ECM database, JSR is used as a cost function to find an optimal point over the ECM technique space for each PAC cycle.

\section{CECM PERformance EVALUATION}

In this section, we present simulation results evaluating the performance of CECM architecture.

For the hybrid ECM memory, the replicator network is composed of an input layer of source nodes, three hidden layers, and an output layer, whose sizes are 8, 10, 5, 10, and 8 , respectively. We call this structure the CECM-hybrid. The weights of the replicator network were initialized as uniformly distributed small numbers on the interval of $(0.125,0.125)$. The learning rate was linearly assigned on the region of $\left[10^{-1}, 10^{-5}\right]$. To train the replicator network, 20 different emitters and 200 training data sets for each were selected. The number of epoch was set as 300 .

The training data set in Fig. 4 was selected from land and air radar emitters which can classified as a threat for a helicopter platform. Then, the real emitter parameters are slightly modified, hence the parameters are restricted.

After training the replicator network, an additional output layer of size 3 was connected to the encoder. The hybrid

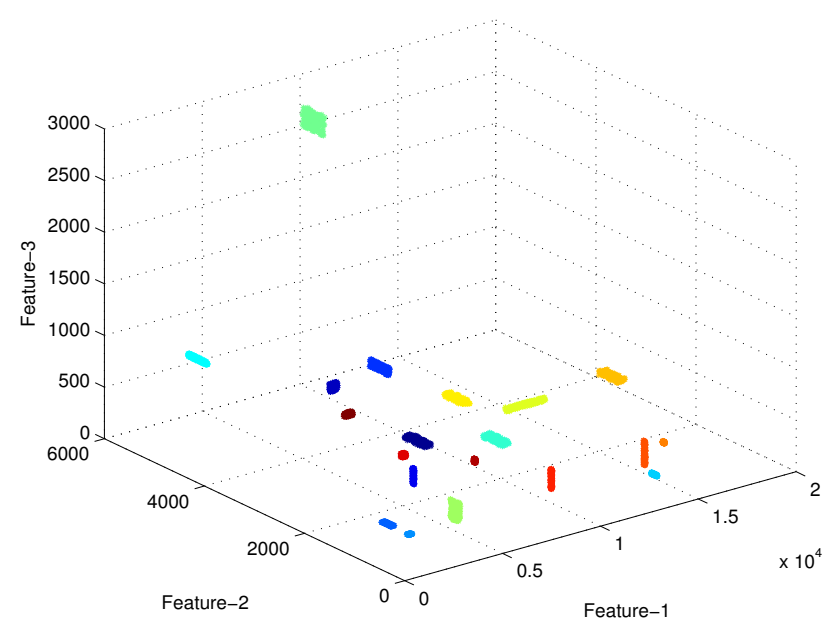

Fig. 4. ECM Memory Training Data Set

network size becomes $8,10,5$ and 3 . The Bayesian regularization algorithm was adopted to train the weights between the encoder and this new output layer. Furthermore, the number of epoch was also set as 300. To train the hybrid network, the same training data sets were selected. The only exception is that this data set contains technique training vectors, which are mapped to each emitter vector. For the technique space, 5 different ECM techniques were used with a 100x100 wide parameter space.

For the multi-layer ECM memory, the network is composed of an input layer of source nodes, three hidden layers, and an output layer, whose sizes are $8,10,5,10$, and 3 , respectively. We call this structure $C E C M-3$. The weights of the multilayer network were initialized as uniformly distributed small numbers on the interval of $(0.125,0.125)$. To train the multilayer network, the same training data sets were selected and the number of epoch was set as 300 .

Finally, we evaluated these two network structure with respect to single hidden layered neural network. The single hidden layer network sizes are 8, 10 and 3. We call this structure as $C E C M-1$.

The early stop mechanism is used in the simulations to avoid over-training. If the network training of mse performance decreased below $10^{-2}$ or the process reached 300 epochs, the operation was terminated immediately.

\section{A. Error Performance}

After training, test vectors are used to evaluate the performance of the training process. Fig. 5 illustrates the error histogram of CECM-3. The less deviated histogram around zero error indicates a successful training process. Before the training operation, data sets were separated and supplied to the network for train, validation and test purposes with $70 \%$, $15 \%$ and $15 \%$ respectively. For all sets, error behaviour shows same performance and small amount of instances reach only maximum $2 \%$ error. 


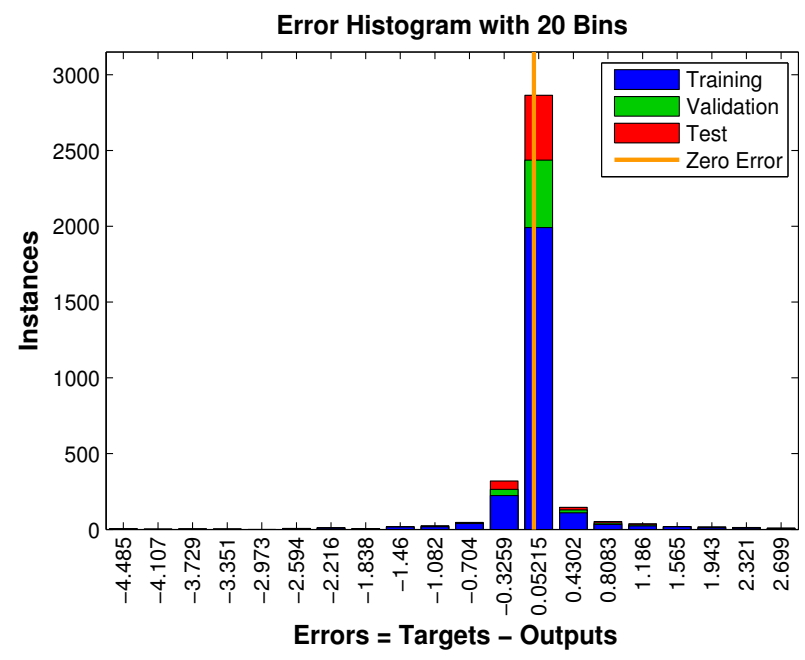

Fig. 5. ECM Memory Error Performance

\section{B. Generalization Performance}

Generalization performance directly reflects the CECM response to unknown emitters. In other words, a more generalized network represents a selection of a more proper ECM technique to unknown emitters.

Fig. 6 displays the generalization performance of different CECM structures as CECM-1, CECM-3 and CECM-hybrid. Generalization performance is evaluated over a mean square error (mse) calculation between target and output vector under different disturbance values,

$$
m s e=\frac{1}{N} \sum_{i=1}^{N}\left(e_{i}\right)^{2}
$$

where $e_{i}$ is the error between target and output vector. Although the CECM-hybrid has the best mse error at zero disturbance, it becomes worst when disturbance increases. CECM-3 has the best generalization performance; hence, it displays almost the same performance under changing disturbance values.

\section{CONClusions And Future Work}

The system scenarios being used in current ECM systems are limited to fixed algorithms which may become obsolete at near future if the number of cognitive radar systems grow in the field. These radars will be capable of sensing the environment and adapting their transmissions to increased performance and overcome interference effects. Therefore, there is an urgent need for a new type of ECM system to overcome the challenges comes from future radars.

Our simulations reveal that CECM with multi-scale memory is a great candidate for next generation ECM systems. Especially, generalization performance indicates that CECM-3 has the best technique selection architecture for unknown emitters which can be classified as threat radar.

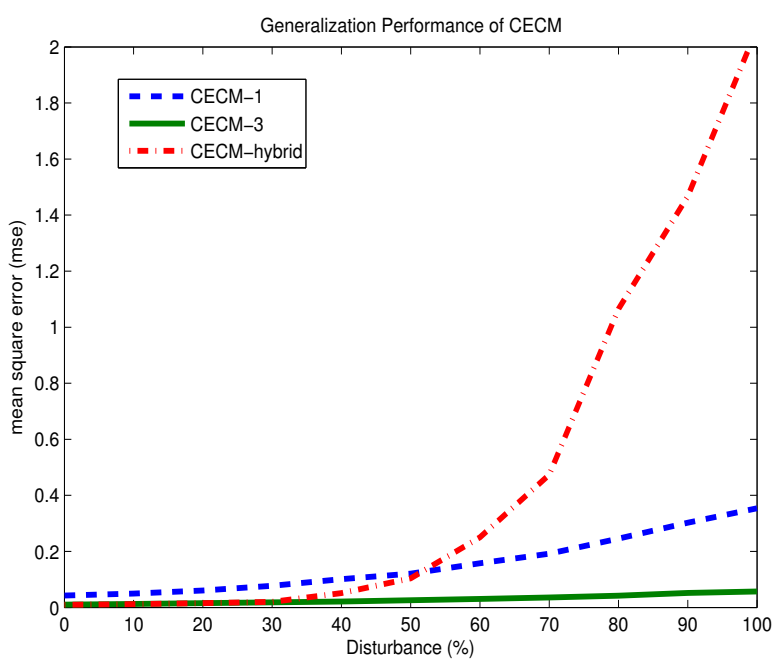

Fig. 6. ECM Memory Generalization Performance

For more real evaluations, CECM assessment with real radar and missile parameters using Tactical Engagement Simulation Software (TESS) [13] and software implementation of the CECM system in a real-time operating system are left as future work.

\section{REFERENCES}

[1] E. J. Chrzanowski, "Active Radar Electronic Countermeasures," Artech House Radar Library, First Edition, December 1990.

[2] B. Friedlander, A. J. Rockmore, "Adaptive control for electronic countermeasures," IEEE Conf. on Decision and Control, vol. 18, pp. 612-623, Dec. 1979.

[3] S. Haykin, "Neural Networks and Learning Machines," Prentice-Hall, 3rd ed. Englewood Cliffs, 2009

[4] MacKay, "Neural Computation," Prentice-Hall, 1992

[5] S. Haykin, "Cognitive dynamic systems: Radar, control and Radio," Proc. IEEE, July 2012

[6] S. Haykin, "Cognitive Radar: Step Toward Bridging the Gap Between Neuroscience and Engineering," Proc. IEEE, Nov. 2012

[7] "Adaptive Radar Countermeasures (ARC)," Information Innovation Office, www.darpa.mil/Our_Work/I2O/Programs/Adaptive_Radar_ Countermeasures_(ARC).aspx

[8] Keith R. Doucet, "Application of Spatial Diversity for Jog Detection in the Presence of Multipath Effects," PhD. Thesis, http://curve.carleton.ca/system/files/theses/23069.pdf

[9] M. Conning, F. Potgieter, "Analysis of Measured Radar Data for Specific Emitter Identification,” IEEE Conf., 2010.

[10] M. Zhu, K. Fu, X. Huang, S. Wu, W. Jin, "A Novel Recognition Approach for Radar Emitter Signals Based on On-Line Independent Support Vector Machines," Advances in Computer Science and its Applications (ACSA), Vol. 2, No. 3, pp. 390-396, 2013.

[11] J. Matuszewski, "Knowledge-Based Signal Processing For Radar Identification," International Journal of Computing, 2008, Vol. 7, Issue 1, 80-87.

[12] F. Dan Foresee, Martin T. Hagan, "Gauss-Newton Approximation to Bayesian Learning," Proceedings of the International Joint Conference on Neural Networks, June, 1997.

[13] “TESS - Tactical Engagement Simulation Software," Tactical Technologies Inc, http://tti-ecm.com/tess.aspx 